\title{
Informalidad como modo generalizado de urbanización: autoetnografía ${ }^{1}$
}

\author{
Informality as a generalized mode of urbanization: \\ an autoethnography
}

Cómo citar:

Muñoz Escalante, M. (2021). Informalidad como modo generalizado de urbanización: autoetnografía. Designia, 8(2), 113-129.

${ }^{1}$ El artículo se origina desde el semillero de investigación Construcciones Ideológicas del Programa de Arquitectura de la Universidad Antonio Nariño en Neiva, perteneciente a la línea de investigación Arte, Diseño y Sociedad del grupo Ciudad, Medio Ambiente y Hábitat Popular. Su objetivo es reflexionar sobre los temas socioculturales que afectan el quehacer urbano para construir una práctica crítica consciente de la realidad actual del país.

* Arquitecto y escritor (Bogotá, 1974). Arquitecto de la Pontificia Universidad Javeriana y M.Arch del Pratt Institute. Profesor investigador desde el 2005. Autor de artículos de teoría, en particular sobre informalidad, la relación entre ciudad y literatura, y la sostenibilidad como ideología, aparte de algunos libros de ficción. Actualmente es coordinador del programa de arquitectura de la Universidad Antonio Nariño en Neiva, y escribe una columna sobre temas de ciudad en La Gaitana Portal. E-mail: munoz.mauricio@gmail.com ORCID: https://orcid.org/0000-0002-2488-2912
Palabras clave:

Informalidad urbana, enseñanza superior, educación artística, identidad cultural, arquitectura, Colombia

Key words:

Urban informality, higher education, art education, cultural identity, architecture, Colombia.

Recibido: 02/07/2020 Aceptado: 06/11/2020

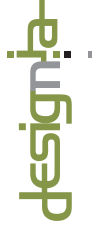

113

\section{Resumen:}

Artículo de reflexión sobre lo que se considera informalidad urbana en la academia colombiana. Se usa la autoetnografía como método de investigación cualitativa. Se cuestiona la idea aceptada de que en la ciudad colombiana coexisten varios tipos de ciudad, siendo la mayoría de ellos diferentes versiones de informalidad. Se arguye que esta atomización de la ciudad, encasillando la informalidad como característica de la pobreza, le permite al teórico 
urbano deshacerse de la responsabilidad. Esto se manifiesta en la creación de una persona, Nosotros, que está por fuera de Ellos, los pares académicos de los países desarrollados, y Ustedes, los habitantes de esas ciudades fuera de todo orden. Se propone que la informalidad es una manera de hacer la ciudad, aun por Nosotros, quienes pretendemos vivir fuera de ella.

\section{Abstract:}

Reflection paper on what urban informality means in Colombian academia. It uses autoethnography as qualitative research method. It doubts the accepted idea of various types of cities coexisting within the same urban center, being the majority of them different versions of informality. It argues that such atomization of the city, pigeonholing informality as a feature of poverty, allows urban theoreticians to avoid their responsibility. This manifests itself in the creation of a person, US, which stands outside of Them, the academic peers of developed countries, and of You, the inhabitants of those out-of-all-order cities. It proposes that informality is a way of making the city, even by $U s$, who pretend to live away from it. 


\section{INTRODUCCIÓN}

En 1961 Lewis Mumford acertó al profetizar que el mundo se convertiría en una ciudad (1979, p. 7). Pero la realidad del capitalismo de esa nueva cultura global no ha permitido que sea una sola ciudad, sino dos, una al norte y otra al sur (Arrighi, 2001). En esa división ya aceptada, el siglo XX fue de las ciudades del Norte Global, y el XXI de las del Sur. Acordemente, según las cifras de la Organización de Naciones Unidas (2018), hasta el 95\% del crecimiento urbano del mundo en las próximas décadas se dará en los países en vías de desarrollo, que están en su gran mayoría en el Sur.

Esto plantea dos paradojas para las ciudades de esos países: que no se cuenta con una hoja de ruta para planearlas, pues muchas de las teorías de cómo funcionan las ciudades están enraizadas en el mundo desarrollado (Roy, 2005); y que la ciudad del Sur Global está dividida internamente en su estudio e impacto (Kudva, 2009).

El artículo parte del clamor de algunos teóricos contemporáneos de no intentar acomodar esas teorías occidentales en sociedades tan diferentes como las del Sur Global $\mathrm{y}$, asimismo, del llamado a estudiar las ciudades que verdaderamente comparten problemas similares en Medio Oriente, África, Sur Asia y Latinoamérica.

Se observa que en Colombia la informalidad se ha convertido en uno de los temas más urgentes de la teoría urbana contemporánea, porque la realidad social y económica ha ido desplazando progresivamente los modelos formales tradicionales, y porque la práctica profesional en contextos cada vez más complejos ha empezado a cuestionar los esquemas usualmente importados de la formación académica en las escuelas de arquitectura y urbanismo del país. Se arguye que el trabajo de conceptualización sobre la informalidad en Colombia ha sido vasto, pero que ha limitado dicha condición a una clase socioeconómica en particular, dejando por fuera las actuaciones de los profesionales y académicos. La hipótesis que se desarrolla en este escrito es que la informalidad urbana no debe ser considerada un fenómeno parcial, exclusivo de ciertos barrios populares, sino una condición total que abarca todos los sectores socioeconómicos de las ciudades de este lado del mundo. 


\section{MÉTODOS}

Se usa la autoetnografía como método de investigación cualitativa. "Auto", pues se conduce desde un punto de vista personal, para estudiar las experiencias propias y aquellas de las comunidades académicas a las que he pertenecido. Se parte de la base de que la autoetnografía adrede valora lo particular como repositorio de una memoria que de otro modo permanecería inaccesible a otras aproximaciones científicas (Chang, 2008). En coherencia con otras orientaciones posmodernas, la autoetnografía asume que el conocimiento se construye desde donde se habla (el lugar) y desde quien lo hace (la identidad) (Ellis, 2009), acogiendo las limitaciones del contexto en vez de ignorarlas.

"Etno", pues el objetivo de la investigación es sacar a flote cómo la cultura da forma y se forma por lo individual (Hall, 2003), donde el desarrollo personal se concibe como construido socialmente. Esto emerge como necesidad en el discurso urbano contemporáneo latinoamericano, que acude de manera recurrente a teorías descoloniales (Rea, 2015; Varley, 2013).

Y "Grafía" (escritura), porque hace énfasis en los aspectos creativos narrativos para generar, documentar y analizar información. Se asume que la escritura es el medio por excelencia para difundir conocimiento. Como puede deducirse de Lawrence (1996), construir una narrativa de un tipo que no sea estrictamente científica permite explorar sentimientos ocultos, motivaciones olvidadas y emociones contenidas, que convierten la actividad de escribir en método de pensamiento.

Se acepta a priori que las memorias y perspectivas personales están mediadas por ideologías que no permiten un acceso transparente a la verdad, pero esto no se observa como debilidad o condición de ingenuidad. Por el contrario, el recurso narrativo puede verse como manifestación de resistencia en contextos donde priman discursos emancipadores.

\footnotetext{
[La autoetnografía] es un texto que emprenden las personas para describirse a sí mismas en maneras que confrontan las representaciones que otros han hecho de ellas. Así, si los textos etnográficos son aquellos en los que sujetos metropolitanos europeos han representado a los otros (usualmente los otros conquistados), los textos auto-etnográficos son representaciones que los llamados otros construyen en respuesta o en diálogo con los primeros (Pratt, 1991, p. 35).
} 
En una geografía de centros y periferias como la que hemos aceptado en América Latina (Floto, 1989), existe un propósito deliberado en el hecho de que un individuo articule sus propias experiencias en lugar de que otros lo hagan por él. En el caso de los teóricos urbanos para quienes la ciudad se analiza primordialmente con base en concepciones de agentes externos "centrales", la autoetnografía se erige como un medio valioso para representar las experiencias y conocimientos "periféricos" de una manera menos amenazante para la academia ya institucionalizada.

En este trabajo se usa la autoetnografía analítica en el sentido de Anderson (2006) y Ellis \& Bochner (2006), que promueven observaciones explícitas dentro de la experiencia.

\section{Se abre el telón}

La noche había sido larga. Los rostros de los estudiantes revelaban jornadas en vela de trabajo incesante. A pesar de sus atuendos de última moda, muchos lucían despeinados y con ojeras, despidiendo tufo de café trasnochado. Unos estaban parados sobre butacas de madera, colgando de las vigas de la cubierta los paneles impresos en papel fotográfico. Otros estaban sentados pegando barandas y personas en sus modelos a escala. Un cigarrillo olvidado en cantiléver sobre una mesa, con su larga ceniza curvada desafiando la gravedad, arrojaba una columna de humo que se enroscaba juguetona hasta llegar al techo.

Una vez estuvo todo puesto en su sitio, nos sentamos a esperar. El jefe de departamento pasó una última vez por los pasillos, acompañado del decano y los coordinadores de área, para verificar que todo estuviera en su sitio. Todo estaba impecable. Si no fuera porque entre los paneles podían entreverse las montañas de la cordillera, sobre los que reposa la ciudad entre el verde andino, cualquiera pensaría que estábamos en alguna escuela europea o norteamericana. 
Trabajaba en la Pontificia Universidad Javeriana (PUJ), hogar de una de las mejores escuelas de diseño del país. Esperábamos la visita de los pares del Royal Institute of British Architects (Riba). La carrera de arquitectura se había postulado para la acreditación internacional de dicho organismo, y ese era «El día». Había televisores proyectando videos en alta resolución, maquetas luminosas, perspectivas futurísticas, computadores portátiles generando imágenes tridimensionales. Todo estaba listo para la función.

Los enviados del Riba caminaron con calma por entre las propuestas. Se detenían brevemente ante ellas y los estudiantes explicaban en inglés casi perfecto sus intenciones. A veces se producía un corto debate, siempre inclinando la balanza hacia el lado de los pichones de arquitectos. Habían logrado una presentación excelsa. Se respiraba un ambiente académico como el que nunca más he tenido la oportunidad de vivir en Colombia. Los profesores del Riba tomaron tinto y comieron empanadas en compañía de sus homólogos locales, y se sentaron a discutir en las bancas como si estuvieran en un taller de la Architectural Association o la escuela Bartlett de su Londres natal.

Cuando llegó el momento de la verdad, nos desplazamos al auditorio perfectamente ordenado. El interior enchapado en madera, la mesa de los panelistas decorada con los colores corporativos, los ujieres limpiamente trajeados, las banderas alineadas al fondo; todo el conjunto inspiraba respeto e institucionalidad.

La exposición se dio en los términos esperados, casi que siguiendo un libreto previamente ensayado, hasta que uno de los profesores invitados rompió el protocolo: «Lo único que me preocupa», dijo, «es para dónde son esas propuestas que vimos».

Un silencio sepulcral se instaló en el lugar. Si alguien hubiera dejado caer una mota de algodón sobre el tapete de fieltro, ésta se hubiera escuchado como un trueno. ¿A qué se refería? ¿Acaso no era obvio? Todos los proyectos se implantaban en lugares específicos de la ciudad, después de cuidadosos análisis urbanos en escalas metropolitanas, sectoriales y barriales. 
Los directivos de la facultad de la PUJ se miraron con desconcierto, hasta que finalmente uno de ellos se acomodó en la silla en señal de responder. Miró el micrófono que sostenía en sus manos y movió el interruptor hacia arriba. Una luz verde se encendió, indicándole que ya podía hablar: «Para Bogotá», dijo.

El profesor inglés agradeció el acto de coraje de su compañero colombiano y siguió con la discusión: «La razón por la que pregunto es porque llegamos hace tan sólo un par de horas y recorrimos la ciudad y en ningún momento vimos nada que se pareciera».

En la presentación que se había preparado para la visita había proyectos que hubieran podido ser diseñados por Zaha Hadid o Herzog \& deMeuron o Rem Koolhaas o David Chipperfield. El hombre del Riba no pudo haberse confundido, más aún a sabiendas de que esos arquitectos tenían profundas relaciones laborales y académicas en Inglaterra. Era una pregunta retórica. Él sabía que ninguna de esas superestrellas dictaba en nuestro país.

Entre el grupo de profesores responsables de asesorar esos proyectos «como de otro mundo», ninguno había diseñado ni construido nada similar en su práctica profesional en Colombia. La gran mayoría de ejercicios estudiantiles se había estructurado con metodologías de investigación proyectiva basadas en la consulta de referentes-la mayoría de las veces internacionales, por inclinación tanto de estudiantes como de docentes - y en especulaciones formales arraigadas en Europa o Estados Unidos. Algunos de los profesores locales eran en efecto curtidos diseñadores y había también jóvenes habilidosos con apenas un par de obras en su haber. Pero podía hacerse la generalización de que el lenguaje urbano de unos y otros se inscribía claramente en una tradición colombiana de geometrías simples, discursos escuetos, composiciones equilibradas y materiales armónicos con su contexto inmediato, lejos de los avezados experimentos que evidenciaban las planchas y maquetas exhibidas en la exposición.

El hombre que proponía la cuestión, adrede había dejado por fuera la discusión sobre la forma. Daba por sentado que forma, lo que se dice forma, podía hacerla cualquier persona en cualquier parte del mundo. La pregunta que ponía sobre el tapete cuestionaba el lugar donde se pondrían esas organizaciones, la teoría que los inspiraba, la población que los habitaría, y la tecnología que los construiría.

La respuesta que elaboramos, errónea a mi juicio, estribó sobre el derecho que tenemos los colombianos de imaginar ciudades que nunca antes hemos hecho. Y digo «errónea» porque la producción de nuevas formas urbanas esquiva las implicaciones que tiene el comentario sobre lo que está fuera de la forma, que en muchos sentidos es lo que nos define. 


\section{Historia de dos - y muchas más - ciudades}

La acotación del profesor británico no constituía un obstáculo para que la escuela de arquitectura de la PUJ recibiera la acreditación internacional del Riba, como en efecto se dio (Pontificia Universidad Javeriana, 2020). Por el contrario, produjo un debate curricular interesante dentro de la facultad sobre cuál debía ser el rol de las universidades nacionales en la interpretación del contexto local. Hoy esa reflexión puede ser todavía más pertinente, pues el "estar fuera de la forma" sigue siendo la imagen unívoca de la ciudad colombiana y latinoamericana «a pesar del aumento en la dinámica económica y mejoría en la situación de pobreza» (Clichevsky, 2009, p. 64).

Esto se puede evidenciar en la narración autoetnográfica anterior. El desconcierto que causó la pregunta se debía a que los representantes del Riba no podían haber vivido de primera mano la informalidad, en el sentido que creemos habitual de la palabra, pues el corto viaje desde el aeropuerto hasta la universidad se había hecho a través de lo que a nuestro juicio es ejemplo de buen urbanismo en Bogotá: la calle 26, el cementerio central, el centro internacional, el parque Nacional. ¿Por qué consideraban entonces la ciudad como informal?

Vista en retrospectiva, la respuesta se alineó con lo comúnmente aceptado de que Ellos ya tienen una idea preconcebida de las ciudades del tercer mundo: una idea que se alimenta por los medios de comunicación, donde con frecuencia se retratan las ciudades colombianas como sucias y desordenadas; una idea que no se cambiaría en una visita pasajera, así nos hubiéramos esforzado al máximo.

Conviene preguntarse entonces si lo que define la ciudad informal son características que solamente identificamos Nosotros, o sea, los profesores e investigadores de las facultades de arquitectura y urbanismo del país. En ese sentido, es pertinente dejar abierta la posibilidad de que dicha informalidad se entienda de una manera diferente. ¿De cuál otra?.

Para responder esa pregunta debemos buscar la falla-en el sentido de grieta, resquicio, fisura-que puede tener nuestro discurso. Porque no se puede negar que aceptamos la existencia de la informalidad urbana. De hecho, el término aparece por primera vez en América Latina, específicamente para explicar la situación en 
Centroamérica (Mejívar \& Pérez, 1989). El problema, a la luz de los acontecimientos posteriores de finales de siglo XX y comienzos del XXI, es la convicción de Nosotros de que «la informalidad es una abominación que debe ser convertida, dislocada o aniquilada» (Kamete, 2013, p. 17). Él describe la situación en África, que no es muy distinta que la de América Latina: en nuestro contexto esto se hace evidente, por ejemplo, cuando excusamos la informalidad como una preconcepción injustificada (Lara, Coulter \& Melis, 2020); o cuando lamentamos que se juzgue nuestro urbanismo con base en paradigmas creados y difundidos a través de procesos de colonización (Hardoy \& Satterthwaite, 1987); o cuando nos ocultamos detrás de que la ciudad de tercer mundo no debe ser igual a la del primero (Kasarda \& Parnell, 1993).

Es claro que en Colombia y América Latina abogamos por la diferencia: reconocemos que en nuestras ciudades existe la informalidad, pero no somos lo suficientemente radicales para elaborar una diferencia perceptible entre Ellos y Nosotros, es decir entre dos grupos perfectamente definidos en lo que a "forma" de la ciudad se refiere. De hecho, estamos produciendo tres grupos: Ellos, de dónde importamos los modelos urbanos (ciudades occidentales desarrolladas la mayoría de los casos); Ustedes, que viven en ciudades que no se parecen a dichos modelos (en asentamientos ilegales y urbanizaciones desreguladas dentro de nuestras ciudades); y Nosotros, que vivimos en un orden especial dentro del caos (en barrios que se parecen a los de Ellos, pero están en las ciudades de Ustedes).

Esta triada Ellos/Ustedes/Nosotros aparece precisamente porque Nosotros partimos de que la informalidad es externa:

Los emigrantes rurales llegan al centro a los inquilinatos, donde comienzan la adaptación a la vida urbana con la consecuente necesidad de trabajo; posteriormente buscan la seguridad de la posesión por lo cual acuden al mercado informal de la periferia donde construyen progresivamente su vivienda; y allí la comodidad de sus viviendas comienza a ser su mayor preocupación (Janssen, 1984, p.181).

En nuestras palabras, los habitantes de la ciudad informal «llegan», «comienzan», «buscan», «acuden», «construyen». Esto nos ubica a Nosotros fuera de dicha condición de informalidad. Nosotros somos observadores de un fenómeno del que no somos parte. Y una vez Ustedes están fuera de nuestra esfera, entonces Nosotros quedamos del lado de Ellos para explicar el fenómeno urbano de la ciudad del tercer mundo: decimos con Bahr \& Mertins (1985) que la deficiente repartición de la riqueza es el origen de la informalidad; revelamos con Turner (1965) el origen no-normativo dentro de la ciudad; establecemos con Deler (1986) las razones de la rápida expansión de la periferia; identificamos con Gilbert (1994) los patrones de vivienda colectiva; explicamos con Rodgers, Beall \& Kanbur (2012) los procesos de reconexión urbana; 
definimos con Castells (1973) la marginalización en el contexto capitalista, etcétera. La ciudad informal de Ustedes la explicamos Nosotros con las teorías de Ellos. Esto limita la condición de informalidad de la ciudad sólo a Ustedes, y facilita que Nosotros les pongamos el mote que tengamos a bien usar-urbanización "irregular", "pirata", "de supervivencia", "ilegal", "fragmentada" - pues Ustedes usualmente no tienen voz.

Esta estrategia nos permite dividir la ciudad en cuántas partes sea necesario: Duhau y Giglia (2008), por ejemplo, dicen que son cinco; y Massida (2019) encuentra diferencias entre tugurio y asentamiento y chabola y favela y comuna y barriada y shanty town y tenement y muchas más. Para Nosotros cada una de esas muchas ciudades tiene un patrón, así sea desconocido. Lo que se nos vuelve de particular importancia es zanjar la diferencia, alejarlas por su misma complejidad:

Conocer objetivamente los procesos de construcción de la ciudad, en este caso la ciudad informal, así como los procesos de producción y transformación de la vivienda y el hábitat para los sectores de población de bajos ingresos, implica tener en cuenta la acción de múltiples actores y sus determinaciones, y reconocer cuáles son los agentes sociales que actúan en ella y cómo intervienen (Torres, 2007, p. 59-60).

Nosotros no somos parte de la ciudad informal porque es de Ustedes, de la «población de bajos ingresos»; la ciudad informal hace parte de otra realidad porque Nosotros vivimos en una economía formal: «la ciudad informal y la economía informal son dos caras de una misma moneda y pertenecen a una sola realidad» (Torres, 2009, p. 41).

El problema aparece porque la correlación que planteamos entre informalidad urbana y pobreza no obliga a que la supuesta ciudad paralela desde donde hablamos Nosotros sea "formal", sino que es menos pobre: decimos que casi el sesenta por ciento de la ciudad colombiana es informal (López, 2018, p. 137), pero no concluimos que el cuarenta por ciento restante sí esté planeado; afirmamos que los efectos del urbanismo sobre la realidad de los procesos de construcción de la ciudad «son bastante limitados y, muy a menudo, contrarios o diferentes a los que se esperaba producir con las acciones y medidas definidas por los planes y otros instrumentos de la planeación» (Cortes, 2007, p. 162-163), pero no nos atrevemos a llamar informales también a estos inesperados resultados de Nosotros. 
No existe idea de "formalidad" dentro la ciudad, porque incluso los procesos conducidos por Nosotros surten efectos no deseados. Podríamos mejor acoger la idea de una sola informalidad, y no de dos ni de tres informalidades externas a Nosotros. No es lógico hablar de varias ciudades de Ustedes que coexisten dentro de la ciudad, y que Nosotros habitamos una ciudad formal desde donde podemos señalar con autoridad qué es informalidad y qué no. La primera condición de la informalidad es precisamente que es de Todos; que «no es sólo un problema politicoeconómico sino una manera de vivir» (AlSayyad \& Roy, 2004, p. 27).

Esa informalidad generalizada se hace evidente en los mismos barrios que Nosotros catalogamos como informales: declaramos que son caóticos porque no hubo profesionales en diseño urbano como Nosotros involucrados en el proceso (Hernández, 2008), pero no podemos explicar las vías y los servicios públicos domiciliarios y las rutas de transporte y los colegios y la infraestructura hospitalaria que paulatinamente aparecen. Cuesta pensar que, si son comunidades tan deprimidas económicamente como decimos, haya habido recursos propios para realizar esos costosísimas trabajos. Lo lógico es que lo mucho o poco que hay lo haya hecho el Estado (Benavides \& Leaño, 2018), a través de sus administradores distritales o locales, quienes a su vez contratan a los planificadores y urbanistas que gradúan las universidades. Y se supone que el Estado está en el lado formal de la ecuación. Eso quiere decir que el Estado opera también de manera informal, para empezar desde su accionar legislativo:

[...] cabe reconocer que el derecho colombiano resulta algo insuficiente a la hora de articular mecanismos de esencia normativa con mecanismos sociales, puesto que su función, centrada en la racionalidad, dificulta la apertura de alternativas acertadas y oportunas que operen de manera eficaz frente al cumplimiento de principios en materia urbanística y en materia de desarrollo territorial (Garcés, 2010, p. 41). 
Dos explicaciones se proveen tradicionalmente a esto: que las intervenciones del Estado-con Nosotros-son después de que la informalidad ya se ha implantado, es decir, que las intervenciones urbanas posteriores se hacen para intentar corregir los errores anteriores de la mejor manera posible (Torres, Rincón, Vargas \& Amaya, 2013), o que la corrupción política y económica juega un papel decisivo en la proliferación de ciertas formas urbanas (Sáenz \& García, 2019). Ambas tienen su parte de verdad, sin embargo, no explican la informalidad sino que la perpetúan: por un lado, el reconocimiento de que los estándares de diseño urbano no son estándares sino que difieren según sea el contexto socioeconómico de los usuarios; y por el otro, la inconsistencia de la legislación, que impulsa a que prosperen distintas formas de ilegalidad bajo el amparo de la misma ley (Fernandes \& Varley, 1998; McAuslan (2002), son ambas muestras de informalidad.

En esos términos, la persona en condiciones de pobreza que se acusa de informal sale de la ecuación. Ésta no actúa fuera de la forma porque no tiene la forma como referente. No detecta forma u orden o armonía en su entorno, al punto de que produzca la reflexión de que su actuación esté fuera de lugar. Al contrario, sus formas están a tono con las otras formas del contexto, en lo social, en lo legal y en lo urbano. Nosotros, que conocemos la forma de hacerlo, pero no lo hacemos, fomentamos la informalidad mientras creemos que hacemos la formal. La informalidad no es pues dominio de los pobres sino que también es importante para las clases medias, e incluso la élite, de las ciudades del segundo y tercer mundo. Estas tendencias apuntan a un incesante complejo de legalidad e ilegalidad [con] diferentes maneras de legitimarse. La división aquí no es entre formalidad e informalidad; más bien se trata de diferencias dentro de la informalidad (Roy, 2005, p. 149). 


\section{CONCLUSIONES}

Ante el inminente crecimiento urbano de las ciudades del tercer mundo en las siguientes décadas, es necesario que los profesionales urbanistas y los académicos en asuntos urbanos tomemos en cuenta los procesos de urbanización típicos de nuestros países, y renunciemos a los intentos de acomodar en ellos teorías y proyectos de ciudades desarrolladas, pues éstos experimentos históricamente han fallado. Reconocernos en la informalidad, que es sin duda la expresión de todos los renglones de la sociedad, puede ser un paso adelante en dicho propósito. La informalidad, entendida como forma de ser y como manera de vivir, y no simplemente como producto diferenciador entre clases sociales, puede convertirse en un modelo convincente para contrarrestar los ideales concebidos por los teóricos de los países desarrollados. Los más sagaces de ellos saben que es la única legítima diferencia: ya decía Koolhaas (2001, p. 653) que Lagos "se está volviendo moderna de una manera "Africana" válida [en el marco] de una modernidad globalizante. Esto para decir que Lagos no nos está alcanzando, sino más bien, que nosotros estamos alcanzando a Lagos». Ya existen indicios sobre esta inversión de la tendencia (Tsenkova, 2012; Kreibich, 1998), lo que abre la posibilidad de que asimismo haya maneras "Medioorientales" y "Surasiáticas" y "Latinoamericanas" de hacer la ciudad. Quizás hoy sea prematuro aún imaginar un mundo sin Ellosy Nosotros, pero podemos evitar la distinción entre Nosotros y Ustedes, en el sentido en el que se usó en este escrito. Parece demasiado fácil decir que la informalidad de las ciudades es culpa de los hombres y mujeres arrojados a las grandes metrópolis por violencia y pobreza rural a vivir en situación de precariedad, y que los planificadores y urbanistas graduados y posgraduados, los profesores y académicos de las escuelas de educación formal como Nosotros, no tenemos nada que ver. Al contrario: tal parece que la informalidad es un «modo generalizado de urbanización» (Roy, 2005, p. 147). 


\section{REFERENCIAS BIBLIOGRÁFICAS}

AlSayyad, N. \& Roy, A. (2004). Urban informality as a 'new' way of life. In Urban Informality: Transnational Perspectives from the Middle East, Latin America, and South Asia, (pp. 7-30). Lanham: Lexington Books.

Anderson, L. (2006). Analytic autoethnography. Journal of Contemporary Ethnography 35, 373-395. Doi: 10.1 $177 / 0891241605280449$

Arrighi, G. (2001). Global capitalism and the persistence of the North-South divide. Science \& Society, 65(4), 469-476.

Bahr, J. \& Mertins, G. (1985). Un modelo de diferenciación socioespacial de las metrópolis de América Latina. Bogotá: Instituto Geográfico Agustín Codazzi.

Benavides, T. \& Leaño, A. (2018). Rol del Estado en los procesos de urbanización periférica de las ciudades colombianas. Margen, 88(1), 1-9.

Castells, M. (1973). Imperialismo y urbanización en América Latina. Barcelona: Gustavo Gili.

Chang, H. (2008). Autoethnography as method. Walnut Creek: Left Coast Press.

Clichevsky, N. (2009). Algunas reflexiones sobre informalidad y regularización del suelo urbano. Bitácora Urbano-Territorial, 14(1), 63-88.

Cortés, R. (2007). Del urbanismo a la planeación en Bogotá (1900-1990). Esquema inicial y materiales para pensar la trama de un relato. Bitácora Urbano-Territorial, 11(1), 160-213. 
Deler, J. (1986). Ciudades andinas: viejos y nuevos modelos. Lima: Instituto de Estudios Peruanos.

Duhau, E. \& Giglia, A. (2008). Las reglas del desorden: habitar la metrópoli. México: Siglo XXI Editores/ Universidad Autónoma Metropolitana, Azcapotzalco.

Ellis, C. \& Bochner, A.P. (2006). Analyzing analytic autoethnography: an autopsy. Journal of Contemporary Ethnography, 35(1), 429-449. https://doi.org/10.1177/0891241606286979

Ellis, C. (2009). Revision: autoethnographic reflections on life and work. Walnut Creek: Left Coast Press.

Fernández, E. \& Varley, A. (Eds.). (1998). Illegal cities: law and urban change in developing countries. Londres: Zed Books.

Floto, E. (1989). El sistema centro-periferia y en intercambio desigual. Revista de la Cepal, 39(1), 147-167.

Garcés, A. (2010). La informalidad urbana y los instrumentos de gestión del suelo. Revista Científica Guillermo de Ockham, 8(1), 39-48.

Gilbert, A. (1994). The Latin American city. London: Latin American Bureau.

Hall, E.T. (2003). Urbe y cultura. En La dimensión oculta (p. 202-221). México DF: Siglo Veintiuno Editores.

Hardoy, J.E. \& Satterthwaite, D. (1987). La ciudad legal y la ciudad ilegal. Buenos Aires: Grupo Editor Latinoamericano.

Hernández, M. (2008). Procesos informales del espacio público en el hábitat popular. Bitácora UrbanoTerritorial, 2(13), 109-116.

Janssen, R. (1984). Vivienda y luchas populares en Bogotá. Bogotá: Tercer Mundo Editores.

Kamete, A. Y. (2013). On handling urban informality in southern Africa. Geografiska Annaler: Series B, Human Geography, 95(1), 17-31.

Kasarda, J. D. \& Parnell, A. M. (1993). Third world cities: problems, policies and prospects. Londres: Sage Publications.

Koolhaas, R. (2001). Lagos: Harvard Project on the City. In R. Koolhaas, S. Boeri, S. Kwinter \& H.U. Obrist (2001). Mutations (pp. 651-719). Barcelona: Actar.

Kreibich, V. (1998). The spatial form of the informal city: Rome, Madrid, Dar es Salaam. Journal of Area Studies, 6(12), 120-134. 
Kudva, N. (2009). The everyday and the episodic: the spatial and political impacts of urban informality. Environment and Planning A, 41(7), 1614-1628.

Lara, J. A., Coulter, C. M., \& Melis, A. (2020). Temporary appropriation and urban informality: exploring the subtle distinction. Cities, 99(1), 102-126.

Lawrence, M.S. (1996). Writing as a thinking process. Ann Arbor: The University of Michigan Press.

López, W. (2018). Diversidad informal urbana, intervenciones particulares para asentamientos específicos. Programas de mejoramiento barrial en Bogotá. Bitácora Urbano-Territorial, 12(2), 135-142.

Massidda, A.L. (2019). Cómo nombrar a la informalidad urbana: una revisión de las definiciones en uso, sus implicaciones analíticas y su alcance. Quid, 16(10), 301-315.

McAuslan, P. (2002). Tenure and the law: The legality of illegality and the illegality of legality. In G. Payne (Ed.). Land, rights, and innovation: Improving tenure security for the urban poor (pp. 22-38). London: ITDG Publishing.

Menjívar, R. \& Pérez, J.P. (1989). Informalidad urbana en Centroamérica: evidencias e interrogantes. FLACSO-Guatemala.

Mumford, L. (1979). La ciudad en la historia: sus orígenes, transformaciones y perspectivas. Buenos Aires: Ediciones Infinito.

Organización de Naciones Unidas. (2018). World urbanization prospects. The 2018 revision. New York: U.N. Department of Economic and Social Affairs. URL https://population.un.org/wup/Publications/Files/ WUP2018-Report.pdf

Pontificia Universidad Javeriana. (2020). Información general. Consultado el 20 de junio de 2020. URL https:// www.javeriana.edu.co/carrera-arquitectura

Pratt, M.L. (1991). Arts of the contact zone. Profession 9(1), 33-40. 
Rea, C. R. (2015). Repensando la relación entre decolonialidad y hegemonía. Nóesis. Revista de Ciencias Sociales y Humanidades, 24(47-2), 39-53.

Rodgers, D., Beall, J., \& Kanbur, R. (2012). Latin American urban development into the 21st century: towards a renewed perspective on the city. New York: Palgrave Macmillan.

Roy, A. (2005). Urban informality: toward an epistemology of planning. Journal of the American Planning Association, 71(2), 147-158.

Sáenz, J.E., García, J.D. (2019). The relationship between corruption and inequality in Colombia: empirical evidence using panel data for the period 2008-2017. Iberoamerican Journal of Development Studies, 8(2):28-43. DOI: 10.26754/ojs_ried/ijds.359

Torres, C.A., Rincón, J.J., Vargas, J.E. \& Amaya, N. (2013). Hacer del mejoramiento barrial y urbano una política pública estratégica para la superación de la pobreza. Bitácora Urbano-Territorial, 23(2), 105-141.

Torres, C.A. (2007). La ciudad informal colombiana. Bitácora Urbano-Territorial, 11(1), 53-93.

Torres, C.A. (2009). Ciudad informal colombiana: barrios construidos por la gente. Bogotá: Universidad Nacional de Colombia.

Tsenkova, S. (2012). Urban planning and informal cities in Southeast Europe. Journal of Architectural and Planning Research, 29(4), 292-305.

Turner, J.F.C. (1965). Asentamientos urbanos no regulados. Caracas: Cuadernos de la Sociedad Venezolana de Planificación.

Varley, A. (2013). Postcolonialising informality. Environment and Planning D: Society and Space, 31(1), 4-22. 